\section{THE PATHOLOGY AND TREATMENT OF} APPENDICITIS.

Abstract of Remarks Made in Introducing a Discussion at the Medical Society of London on Monday, January 22nd, 1900.

By C. B. LOCKWOOD, F.R.C.S., Assistant Surgeon and Lecturer on Surgical and Applied Anatomy, st.

Mr. Lockwood began by referring to the discussion which had already taken place before the Medical Society of London on the Prognosis of Appendicitis and Notes of 200 Cases. Dr. Cayley in his very thoughtful communication, and some of those who spoke, had to a large extent made use of clinical statistics. This method of inquiry was obviously of value, but had not hitherto carried conviction to the minds of the physicians and surgeons. The reason for this was simple. Taking Dr. Cayley's class of 99 cases of simple appendicitis and perityphlitis which were said to have recovered it was reasonable to ask how this was known to be true. A certain proportion of them must have had concretions in the appendix. The attack might have subsided. But they had not recovered, and the appendicitis was sure to recur. It would be just as reasonable to say that a patient with stone in the bladder had recovered because after rest in bed an attack of cystitis had subsided. Without doubt some of the 99 cases relapsed and went to other hospitals and were there included amongst the severe cases with perforation, gangrene, or septic peritonitis.

Mr. Lockwood ventured to think that appendicitis could not be correctly prognosed until it was possible to infer from the clinical symptoms the pathological changes proceeding within the appendix. He did not intend to discuss the nomenclature of diseases of the vermiform appendix. Much confusion prevailed, and the classification adopted by many authors was illogical. When the inflammation originated in the vermiform appendix he intended to speak of appendicitis, and add terms to indicate the effects the disease had produced, such as ulceration, perforation, gangrene, the various inflammations of the peritoneum, of the broad ligament of the uterus, of the portal vein, and so forth. Mr. Lockwood's remarks were based upon histological examination of 53 cases of appendicitis, ranging from the slightest to the most acute. The specimens had been obtained from cases which he himself had operated upon, and of which he knew the histories. Mr. Lockwood proposed to illustrate his remarks with a few typical photomicrographs made from the histological specimens of these cases. He felt deeply indebted to Mr. Ernest Shaw for the beautiful sections which he had made, and to Mr. Albert Norman for his exquisite photomicrographs. It was needless to say that the specimens, after hardening in Müller's fluid,were cut in paraffin, and stained and mounted after the best modern methods.

Mr. Lockwood began by referring to the group of cases in which suppuration was absent. Some of these came under the head of appendicular colic. In these, so far as was known, no clear attack of appendicitis had ever occurred. They were characterised by slight tenderness on pressure over some part of the iliac fossa, by disorders of digestion, and by occasional attacks of transient pain, not infrequently mistaken for renal colic. Before placing on the screen the photograph of the sections of such a case as this, photographs of the normal anatomy of the appendix were shown. Special attention was directed to the lymphoid follicles and the lymphatic system of the appendix. The continuity of the submucous and subperitoneal tissues through numerous gaps (hiatus muscularis) in the muscular coats was pointed out. and the paths by which inflammatory and infective inflammations of the mucosa spread to the peritoneum were demonstrated.

In a case of appendicular colic, such as described by Talamon, a healthy young woman suffered for eighteen months from abdominal pains. During the last six months she had been unable to follow her occupation. The pain was referred to the right side of the abdomen and to the right iliac fossa. It had been diagnosed as appendicular colic. The appendix was removed. To the naked eye it appeared normal. Microscopical seclion showed that the lumen was filled with epithelium mucus, granules, and crystalline bodies. This fluid was crowded with cocci, diplo- cocci, small ovoid bacilli, single and in pairs, small clumps of staphylococci, and streptococci in chains from 4 to 6 , also some long slender bacilli. In the photomicrograph the mucosa of the appendix was seen to be ulcerated, and these various bacteria had penetrated for perhaps half a millimetre into its substance. One might be reluctant to advise the removal of the appendix for a disease designated appendicular colic. Such a designation conveyed but little idea of danger. Few would hesitate to remove an appendix the mucosa of which was ulcerated and invaded by bacteria. Other photomicrographs illustrating so-called appendicular colic were shown.

Catarrhal appendicitis was next referred to. Amongst the cases which had been included under this term until the result of the histological examination had been ascertained, the following had been considered a typical case of catarrhal appendicitis. A young gentleman, aged 29, had his first attack of appendicitis at the age of 19 . During the next ten years he had so many attacks that he could not recall the number. They usually confined him to bed for a day or two, and prevented him from going to business. Perhaps an operation had been deferred because he suffered from mitral disease. The appendix was removed in the usual way, and was not adherent. It was engorged with blood and felt very hard. It was constricted about three quarters of an inch from the intestinal end. The photomicrograph showed that the mucosa was ulcerated, that the lumen was filled with granules, crystallinebodies, shreds of organic matter, irregular pieces of semi-transparent substance, and quantities of bacteria. These include many morphological varieties: Cocci, diplococci, short ovoid bacilli, single and in pairs, long slender bacilli, short thick bacilli with rounded ends, long thick bacilli, leptothrix and spores, and spore-bearing bacilli. Many of the tubular glands had shed their epithelium, which had been replaced by plugs of bacteria projecting from the lumen. In some places these bacteria were penetrating the lymph canulicular system of the mucosa. The term catarrhal appendicitis did not perhaps convey to the mind a very clear sense of impending danger, but no one who was aware of the pathological state of such an appendix as this would hesitate for a minute in advising its removal.

Some of the other pathological conditions associated with clinical signs of appendicitis were next illustrated; the effects of stenosis being taken first. The appendix of a young woman who had had four attacks of appendicitis was removed. During the attacks the temperature rose. She had pain in the right iliac fossa, which was so severe as to cause collapse, with constipation, but without vomiting. Between the last two attacks pressure in the iliac fossa was painful, so that it was obvious that although appendicitis had subsided it had not got well. The appendix was not adherent, was nearly 4 inches long, and constricted an inch from the cæcum. The constricted part was shown in the photomicrograph. The mucosa beyond the constriction still possessed an epithelial lining. The lumen contained pus full of streptococci. It was hardly necessary to point out the danger of this condition, but to render it clearer, photomicrographs of another case were shown. A man had had six attacks of appendicitis and was advised to have his appendix removed. The operation was deferred until after his wife had been confined. When next seen he had no signs whatever of appendicitis, but was obviously suffering from hepatic abscess. An attempt was made to discover this abscess from behind, but without success. A second attempt was made in front. No abscess could be discovered, but inflamed glands were felt about the cæcum. The appendix was therefore removed. The photomicrograph showed that the interior contained pus full of streptococci with a few bacteria of other types. The lymph on the surface of the appendix was also full of streptococci. The patient survived this operation, sut ultimately died of portal pyæmia with a number of abscesses in the liver. The delay in this case was evidently a matter for the keenest regret, and would never have been permitted bad the pathological condition of the appendix been accurately diagnosed. Some of the effects of ulceration of the mucosa and subsequent infection of the appendicular wall were next illustrated. Also the dangers of fæcal concretions. Fæcal concretions were shown to be bacterial masses, a point which, strangely enough, had not been demonstrated by any investigator. The speaker regretted 
that he had not time to demonstrate the various steps in the formation of fæcal concretion. These specimens showed that fæcal concretions were always associated with general alceration of the mucosa ; that the concretion was not necessarily the cause of the ulceration; that the concretion and the ulceration both owed their origin to the same cause-bacterial accumulation within the appendix. Specimens were shown to illustrate this point. The presence of concretion in the appendix was evidence of extensive ulceration of the mucosa and bacterial invasion. A large concretion was in itself a mechanical peril when owing to the application of cold to the surface of the body the appendix became engorged. It might cause a perforation of the appendix in its vicinity, or gangrene of the part beyond.

If it were true that many of the cases of appendicitis which were said to have recovered did as a matter of fact contain concretions, it was easy to imagine their peril.

The so-called process of natural cure was next mentioned. The accounts given by authors were fanciful. It was impossible to say when the so-called process of natural cure had been achieved. The appendix had been removed from a man who had had at least twenty attacks of appendicitis. He had twice been in large London hospitals, once under a surgeon and once under a physician. As they sent him out again without operation it was reasonable to suppose that they anticipated that the natural process of cure would eventually take place. The appendix was removed. A histological examination showed that the mucosa had been destroyed by ulceration, and that the lumen was occupied by a concretion which seemed calculated to prevent any supposititious natural cure from taking place. An appendix which had once been inflamed might inflame again after long intervals. An appendix had recently been removed for repeated and violent attacks of appendicitis, which had recurred after a period of quiescence of nine years. Another case had a violent attack of appendicitis, then an interval of fifteen years' quiescence, followed by another violent attack, after which the appendix was removed. Il did not seem reasonable to lay down rules as to how many attacks should be suffered before the appendix was removed. Mr. Lockwood was inclined to wait for some time after the first attack of non-suppurative appendicitis, and see what supervened. But on several occasions the appendix had been removed after the first attack because it was still painful and could be felt. Under these circumstances the general health of the patient was seldom good, and the functions of the alimentary tract were improperly performed, indigestion, flatulence, constipation, and diarrhœe being frequent accompaniments. After a second attack of non-suppurative appendicitis most patients preferred to part with an organ which appeared to be of no physiological importance and when diseased an actual source of danger.

The question of diagnosis was next referred to. It is usually possible to say that the appendix was inflamed, but hitherto the histology of appendicitis had hardly been sufficiently investigated for anybody to surmise with accuracy the actual pathological state of the appendix. The usual sequence of events, as shown by the histological examination of 53 specimens, was destruction of the epithelial lining of the lumen, ulceration of the mucosa, bacterial invasion of the mucosa and submucosa, extension of the inflammation through the muscular gaps to the peritoneum. This process might be complicated by the formation of cicatricial strictures, by inflammatory obliteration of the whole lumen after removal of the mucosa, by the presence of fæcal concretions, by perforation, by gangrene, by inflammation of the appendicular lymphatics, and by portal pyæmia. It was also important to note, as Clado was the first to point out, that the inflammation in females not infrequently spreads along the lymphatics to the right broad ligaments of the uterus, so that appendicitis was sometimes mistaken for inflammation of the ovary or Fallopian tube. Three cases of this kind had been operated upon. In addition, it was important to observe that appendicitis sometimes occurred as a complication of other diseases. Primary tuberculosis of the appendix was probably a rare disease. Although every specimen had been carefully examined for tubercle it had but once been discovered. But on one occasion in operating for the removal of an inflamed appendix general tuberculosis of the peritoneum was met with, the inflammation of the appendix being a complication of that disease. In another case of appendicitis a hard mass was found at the base of the appendix. When a portion of this was submitted to histological examination it was found to be cancerous. The cancer, which involved the ileocæcal valve, was removed, and the intestine united by circular enterorrhaphy. The patient recovered, but ultimately died of a recurrence of the cancer in the pelvis. As regarded cases of appendicitis in which perforation or gangrene had occurred, there could be little doubt as to the propriety of evacuating and draining the abscess as soon as diagnosis could be effected. The cases showed that the safety of the patient depended mainly upon the stage at which the operation was performed, and something, too, upon the position which the appendix occupied. When the appendix was beneath the cæcum, and in the iliac fossa, the chances of the abscess being localised were fair. Likewise when the appendix lay to the outer side of the cæcum and colon. But in females especially the appendix often hung over the brim of the pelvis into Douglas's pouch, when such a one perforated or became gangrenous the danger was vastly increased. Moreover, collections of pus at the bottom of Douglas's pouch were most difficult to drain.

As regarded the operation, Mr. Lockwood advocated the oblique incision parallel to the outer half of Poupart's ligament, because it could be extended to reach an appendix which lay outside the cæcum, or which was adherent within the pelvis. In non-suppurative cases the appendix might be most difficult to discover. It might be laid down as an axiom that the organ was never absent except as the result of disease. Cases were mentioned in which the organ had only been discovered with the greatest difficulty because it was hidden away in either the subcæcal or the ileocæcal retro-peritoneal fossa. Doubtless surgeons who had failed to find the appendix had not infrequently been deceived by this abnormality.

In cases complicated with suppuration and peritonitis a determined endeavour should be made to remove the appendix. It was worth while to run considerable risks rather than leave behind an appendix which was profoundly septic and might contain concretions. In some cases in which the abscess had merely been opened and drained and the appendix left behind, subsequent attacks of appendicitis have been known to occur. In others a septic sinus had persisted re. quiring a formidable and ofttimes fatal operation for the removal of the appendix. In one such fatal case the sinus led to a perforated appendix which contained a large concretion. Nevertheless, the patient's condition sometimes forbade an attempt to remove the appendix. A case of this kind was under observation in which the origin of the appendicular abscess had been followed at intervals by three others of the most formidable and dangerous type. As regarded the closure of the wounds after the removal of the appendix in cases without suppuration, it had been found inadvisable to insert buried sutures of silk, because infection was likely to occur when the lumen of the appendix was cut across. Good results were obtained with a single row of fishing.gut sutures. The wound seldom exceeded 3 inches in length, 2 to $2 \frac{1}{2}$ inches being common.

Operations for the removal of the appendix varied greatly in their gravity. The simple operation was comparable to the radical cure of non-strangulated inguinal hernia, but occasionally, after violent appendicitis, the most formidable adhesions were met with, and might test all the courage and dexterity of the operator before they were overcome.

Mr. Lockwood's cases of operation for the removal of the vermiform appendix, in which suppuration was absent, had recovered more quickly since they had lain upon their side, and quickly been put on solid food. In cases of appendicitis with perforation or gangrene and acute abscess, the aftertreatment required the greatest skill and watchfulness.

The $X$ Rays in Life Insurance Examinations.-F. H. Williams (Boston Medical and Surgical Journal, December 28th, 1899) points out that the chief organs for examination in life insurance are the kidneys, lungs, and heart, and that the question is not so much what the disease is as whether or not there is an abnormal condition of the chest. By using the fluorescent screen, tuberculous foci, emphysema, thoracic aneurysm, or enlarged heart can be visibly demonstrated. 